\title{
Social isolation and predator scent tests alter brain BDNF levels differentially according to gender, in rats and effects of fluoxetine
}

\author{
Aslı Aykaç 1, *, Şule Öncül ${ }^{2}$, Rüş̧tü Onur ${ }^{3}$ \\ 1 Department of Biophysics, Faculty of Medicine, Near East University, Lefkoşa, Turkish Republic of Northern \\ Cyprus \\ 2 Department of Biophysics, Faculty of Medicine, İstanbul Medeniyet University, İstanbul, Turkey, \\ 3 Department of Pharmacology, Faculty of Medicine, Near East University, 99138, Lefkoşa, Turkish Republic of \\ Northern Cyprus \\ * Correspondence: aykacasli@yahoo.com, asli.aykac@neu.edu (A.A.); Tel: +90-392-675 10 00; Fax: +90-392-680 20 00; \\ ORCID No: 0000-0002-4885-5070.
}

Received: 29 September 2017; Revised: 19 December 2017; Accepted: 23 December 2017

ABSTRACT: We compared different stress conditions on brain BDNF levels, in rats exposed to social isolation test (SIT) and predator scent tests (PST). BDNF expression in the frontal cortex, hippocampus, and amygdala was compared, and effects of chronic fluoxetine (FLU) treatment were evaluated. Rats were exposed to SIT and PST for one month. FLU was given $(5 \mathrm{mg} / \mathrm{kg} /$ day, ip) throughout stress procedures. Controls, stress, and treatment groups were evaluated in elevated plus maze, anxiety scores were calculated. BDNF expression was determined by Western blot. SIT and PST induced anxiety in both sexes, females had greater anxiety scores than males $(p<0.05)$. FLU restored anxiety scores in both sexes $(p<0.01)$ in both tests. Male and female rats exhibited reduced cortical BDNF levels in SIT $(p<0.001)$. PST reduced cortical BDNF in females, but increased in males. Hippocampal BDNF expression was lowered in SIT $(p<0.01)$ and PST $(\mathrm{p}<0.001)$ in both sexes. Female rats had $40 \%$ lower BDNF expression than males in the amygdala in SIT. FLU did not restore cortical BDNF in females in both tests, but reduced increased BDNF levels in males in PST ( $<<0.001)$. FLU did not restore reduced brain BDNF in males in the hippocampus and amygdala, but restored in hippocampus, in females. Our findings indicate that sex differences must be considered in studies related to mood disorders of animal models, and suggest that BDNF expression in different brain regions are altered differentially in a gender-dependent manner in rats. Antianxiety effect of FLU is not mediated through increasing BDNF activity in cortex in both genders. Increased BDNF in hippocampus and amygdala may reflect antidepressant effect of FLU in female rats, but not in males.

KEYWORDS: Fluoxetine; BDNF; predator scent test; social isolation test; hippocampus.

\section{INTRODUCTION}

Biological reactions to mental or emotional adverse stimuli disturb psychological homeostasis, and this may lead to mood disorders. Stress gives rise to anxiety and anxiety in turn, may lead to (precipitate) depression. In the pathogenesis of stress-related mood disorders such as major depressive disorder and posttraumatic stress disorder (PTSD), brain-derived neurotrophic factor (BDNF) plays a vital role among other factors. The role of BDNF in depression is widely accepted, because pre-clinical and clinical studies provided evidence suggesting that reduced BDNF expression is involved in pathophysiology of depression [1-3]. Under pathological conditions, expression of BDNF in the hippocampus, limbic system and basal forebrain are reported to be reduced.

Although the human disorder is not identically mimicked in animals, there are several experimental stress-induced models of depression. Exposure of the animals to emotional or physical stress causes depression-like behavior. In these models, BDNF levels and its mRNA expression are reported to be lower in the blood and certain brain areas [2,4-9]. In some experimental models, BDNF is reported to provide protection against stress-induced neuronal damage [10,11], and infusion of BDNF into the midbrain showed an antidepressant-like effect [12,13]. Animal experiments also showed that antidepressant treatments increased the expression of BDNF, especially in the hippocampus [2,14,15].

How to cite this article: Aykaç A, Öncül Ş, Onur R. Social isolation and predator scent tests alter brain BDNF levels differentially according to gender, in rats, and effects of fluoxetine. Marmara Pharm J. 2018; 22 (2): 190-198. 
The neurotrophin hypothesis suggests that since stress and depression are associated with decreased expression of BDNF, antidepressants reduce depressive behavior by increasing BDNF levels in the brain $[1,2,16]$. When selective serotonin reuptake inhibitors (SSRI), such as fluoxetine (FLU) is used, BDNF expression increases in different brain regions [17,18]. Behavioral response to stress displays gender differences. Epidemiological studies indicate that women are more susceptible to stress-induced psychopathologies than men and depression occur twice as frequently in women [19-21].

Stress affects BDNF levels in various brain regions and thus an increased stress response in females could be responsible for the increased incidence of depression in females [22]. In rats who were exposed to chronic psychosocial isolation stress, BDNF mRNA levels were depressed to a similar extent in hippocampus and the chronic FLU treatment increased BDNF mRNA in females but not in males [23].

In this study, we used two different stress conditions, social isolation and predator scent tests and tried to evaluate if these conditions exerted discriminatory effect on anxiety levels and altered brain BDNF levels differentially in the male and female rats. Moreover, the effectiveness of chronic FLU treatment was tested in both genders and also the BDNF expression in the hippocampus, frontal cortex and amygdala were compared.

\section{RESULTS}

We observed that SIT and PSTs effectively induced anxiety in both male and female rats. Female rats had greater anxiety scores than that of male rats in the SIT (Figure 1a, $\mathrm{p}<0.05$ ), as well as in the PST (Figure 1b, $\mathrm{p}<0.05)$. Administration of FLU alone $(5 \mathrm{mg} / \mathrm{kg})$ did not appreciably alter anxiety indexes in both stress tests and both genders. Chronic FLU treatment restored anxiety scores of rats towards control values and the anxiety indexes were found to be significantly lower from that observed in the presence of stressors for both female $(p<0.01)$ and male $(p<0.001)$ rats in two settings.

Female rats had significantly greater basal BDNF density in the frontal cortex than male rats that were exposed to SIT (Figure 2a, $\mathrm{p}<0.01$ ). However, this difference was not observed in the animals that were exposed to PST. When their BDNF levels in their frontal cortex in response to stressful stimuli were compared, female and male rats displayed a different pattern. Female rats exhibited a significant reduction in BDNF levels when they are exposed to both SIT $(\mathrm{p}<0.001)$ and PST (Figure $2 a$ and $2 b, p<0.001)$.
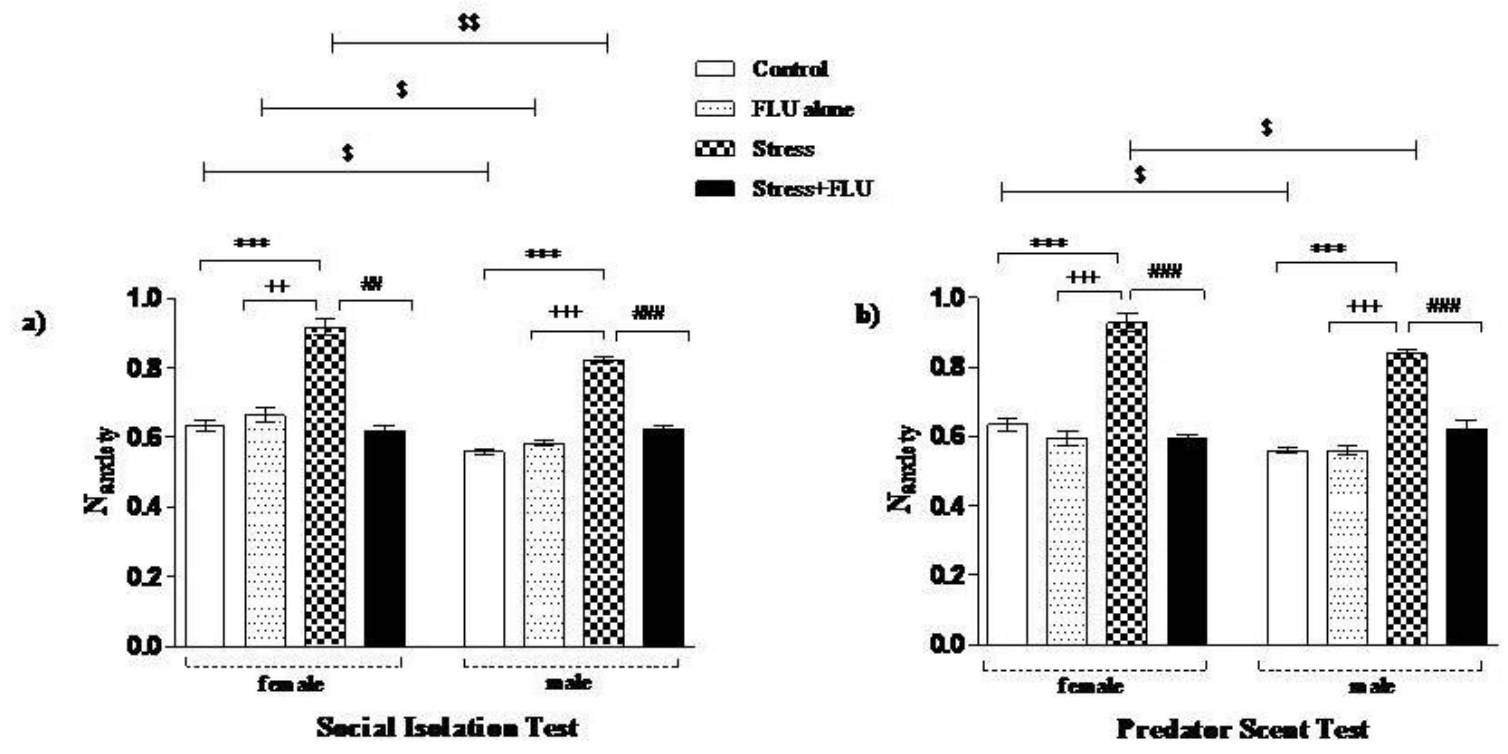

Figure 1. The effects of social isolation test (SIT, a) and predator scent tests (PST, b) on anxiety indexes in the rats and effects of fluoxetine (FLU) treatment. Rats received i.p injection of FLU, $5 \mathrm{mg} / \mathrm{kg} /$ day or saline for 1 month. Anxiety of the animals were evaluated by using elevated plus maze (EPM) before and on the last day of drug/saline treatment. ( $\mathrm{n}=8$ for each group).

${ }^{*}, \mathrm{p}<0.05 ;{ }^{*+*}, \mathrm{p}<0.001$ shows the difference between the stress groups and the control group. ${ }^{++}, \mathrm{p}<0.01 ;{ }^{+++}, \mathrm{p}<0.001$ represents the differeence treatments between FLU alone and stress groups. ${ }^{\#}, \mathrm{p}<0.01 ;{ }^{\neq \neq}, \mathrm{p}<0.001$ shows the difference between stress and stress+FLU groups. $(\$, p<0.05 ; \$, p<0.01)$ represent the difference between the sexes. The data are expressed as means \pm SEM. 
Similar to females, male rats also exhibited a significant reduction in cortical BDNF levels when they were exposed to SIT (Figure 2a, p<0.001). However, PST did not reduce cortical BDNF levels, but an increase was noted in the male rats (Figure $2 b, p<0.05$ ). Chronic FLU treatment did not increase anxiety-induced decrease in cortical BDNF levels in the female and male rats in the SIT. However, FLU significantly (Figure $2 b, p<0.001)$ reduced increased BDNF levels in the male rats in the PST.
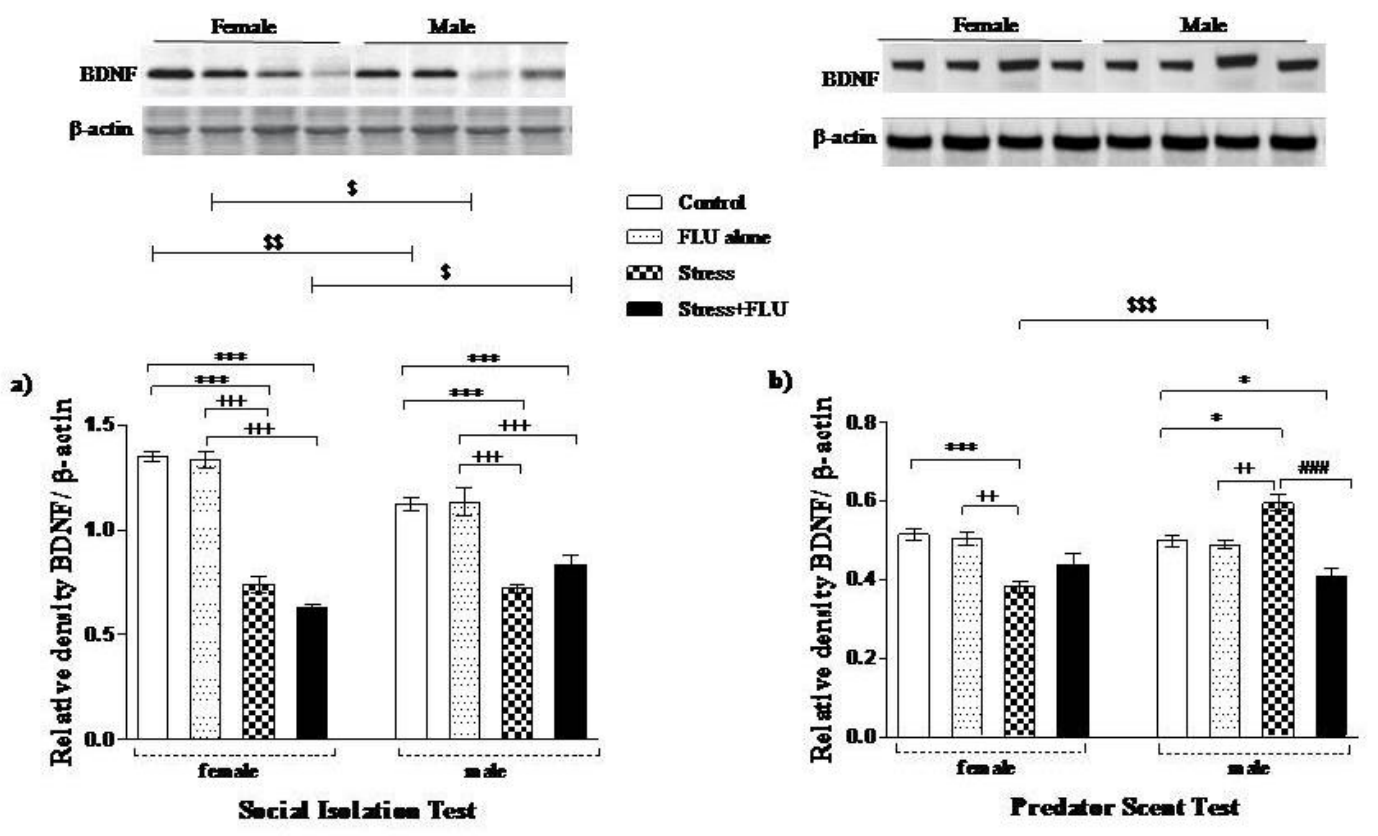

Figure 2. The effects of social isolation test (SIT, a) and predator scent tests (PST, b) on brain derived neurotrophic factor (BDNF) in the frontal cortex in the rats and effects of fluoxetine (FLU) treatment. Rats received i.p injection of FLU, $5 \mathrm{mg} / \mathrm{kg} /$ day or saline for 1 month. Anxiety of the animals were evaluated by using elevated plus maze (EPM) before and on the last day of drug/saline treatment ( $n=8$ for each group). The representative photographs of membranes collected from immunoblotting experiments of rats subjected to SI (a) or PSTs (b) treated with or without FLU. For the immunoblotting analysis $n=4$ rats were used for each group and the analyses were performed as duplicate. $\beta$-actin was used to normalize the amount of protein loaded in each lane.

${ }^{*}, \mathrm{p}<0.05 ;{ }^{*+*}, \mathrm{p}<0.001$ shows the difference between the stress groups and the control group. ${ }^{++}, \mathrm{p}<0.01 ;{ }^{+++}, \mathrm{p}<0.001$ represents the differeence treatments between FLU alone and stress groups. ${ }^{\neq \neq}, \mathrm{p}<0.001$ shows the difference between stress and stress+FLU groups. ${ }^{\$}, \mathrm{p}<0.05 ; \$ \$, \mathrm{p}<0.01 ; \$ \$ \$ \mathrm{p}<0.001$ represent the difference between the sexes. The data are expressed as means \pm SEM.

Hippocampal BDNF levels of rats were similar in both groups before exposure to stressors. SIT and PST significantly reduced hippocampal BDNF expression in both sexes (Figure 3a and b, $\mathrm{p}<0.01$ for SIT, $\mathrm{p}<0.001$ for PST). FLU treatment restored stress-induced reduction in BDNF expression in the female rats in both tests $(\mathrm{p}<0.05$ and $\mathrm{p}<0.01$, respectively). Male rats exhibited a different pattern from that observed in the female rats in their response to FLU treatment. In the male rats FLU treatment did not restore hippocampal BDNF levels in both the PST and the SIT.

When the stressor-induced alterations in BDNF expressions were compared in the amygdala, male and female rats exhibited a different pattern. We observed that female rats had about $40 \%$ lower BDNF expression than the male rats exposed to SIT. This difference was not observed in the PST. SIT reduced BDNF expression in the female rats $(p<0.001)$ but did not reduce it when the PST was applied. However, BDNF expression in the male rats, was not altered in the SIT but was significantly (Figure $4 b, p<0.05$ ) reduced in the PST. FLU treatment restored stressor-induced reduction in BDNF expression in the females in SIT (Figure 4a, $\mathrm{p}<0.05$ ). In the male rats, FLU treatment did not influence BDNF levels in both experimental settings. FLU administration alone did not alter brain BDNF levels in this experimental setting in all brain regions and all genders. 

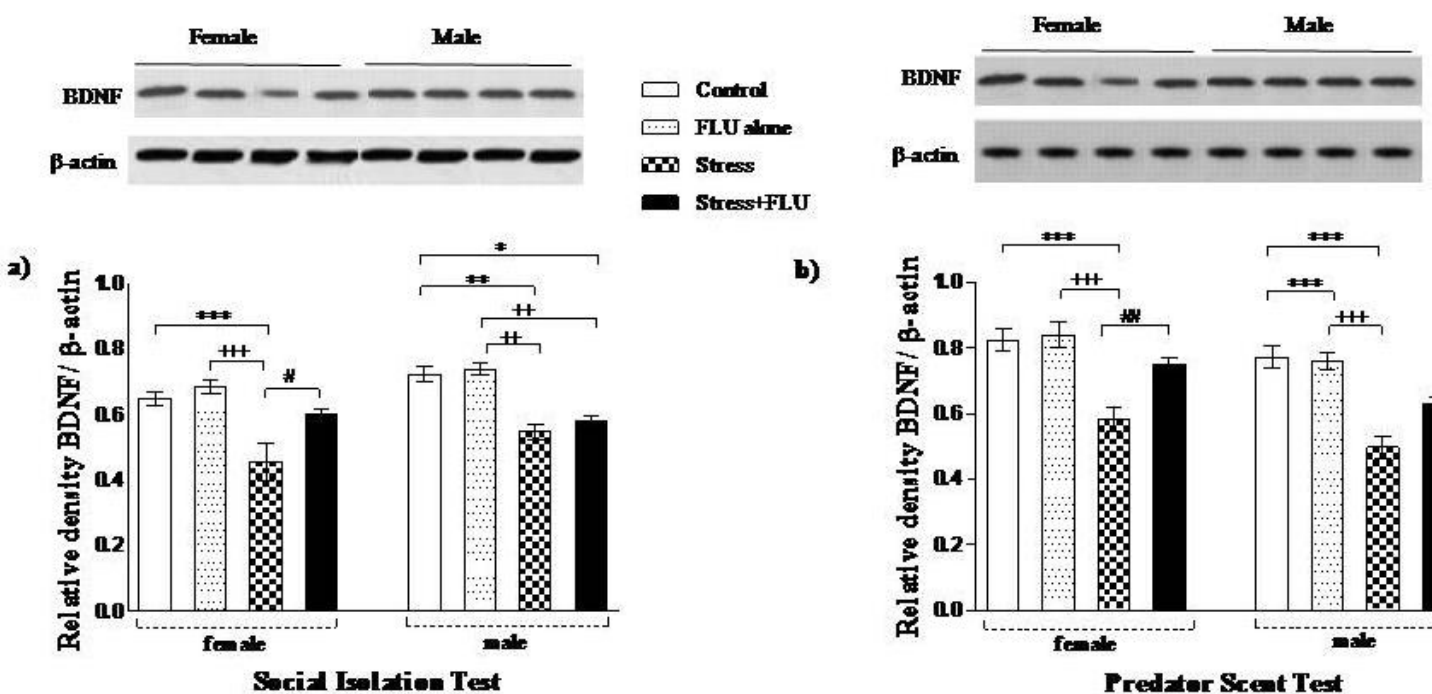

b)

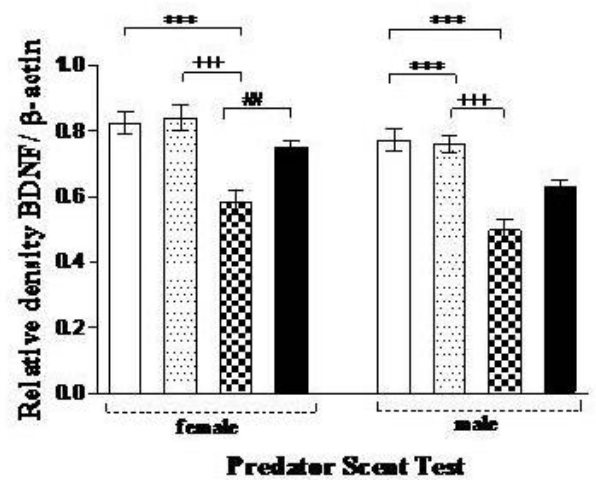

Figure 3. The effects of social isolation test (SIT, a) and predator scent tests (PST, b) on brain derived neurotrophic factor (BDNF) in the hippocampus in the rats and effects of fluoxetine (FLU) treatment. Rats received i.p injection of FLU, 5 $\mathrm{mg} / \mathrm{kg} /$ day or saline for 1 month. Anxiety of the animals were evaluated by using elevated plus maze (EPM) before and on the last day of drug/saline treatment ( $n=8$ for each group). The representative photographs of membranes collected from immunoblotting experiments of rats subjected to SI (a) or PSTs (b) treated with or without FLU. For the immunoblotting analysis $n=4$ rats were used for each group and the analyses were performed as duplicate. $\beta$-actin was used to normalize the amount of protein loaded in each lane.

${ }^{*}, \mathrm{p}<0.05 ;{ }^{*+}, \mathrm{p}<0.01 ;{ }^{* *+}, \mathrm{p}<0.001$ shows the difference between the stress groups and the control group. ${ }^{++}, \mathrm{p}<0.01 ;{ }^{+++}, \mathrm{p}<0.001$ represents the differeence treatments between FLU alone and stress groups. ${ }^{\# \neq}, \mathrm{p}<0.001$ shows the difference between stress and stress+FLU groups. The data are expressed as means \pm SEM.
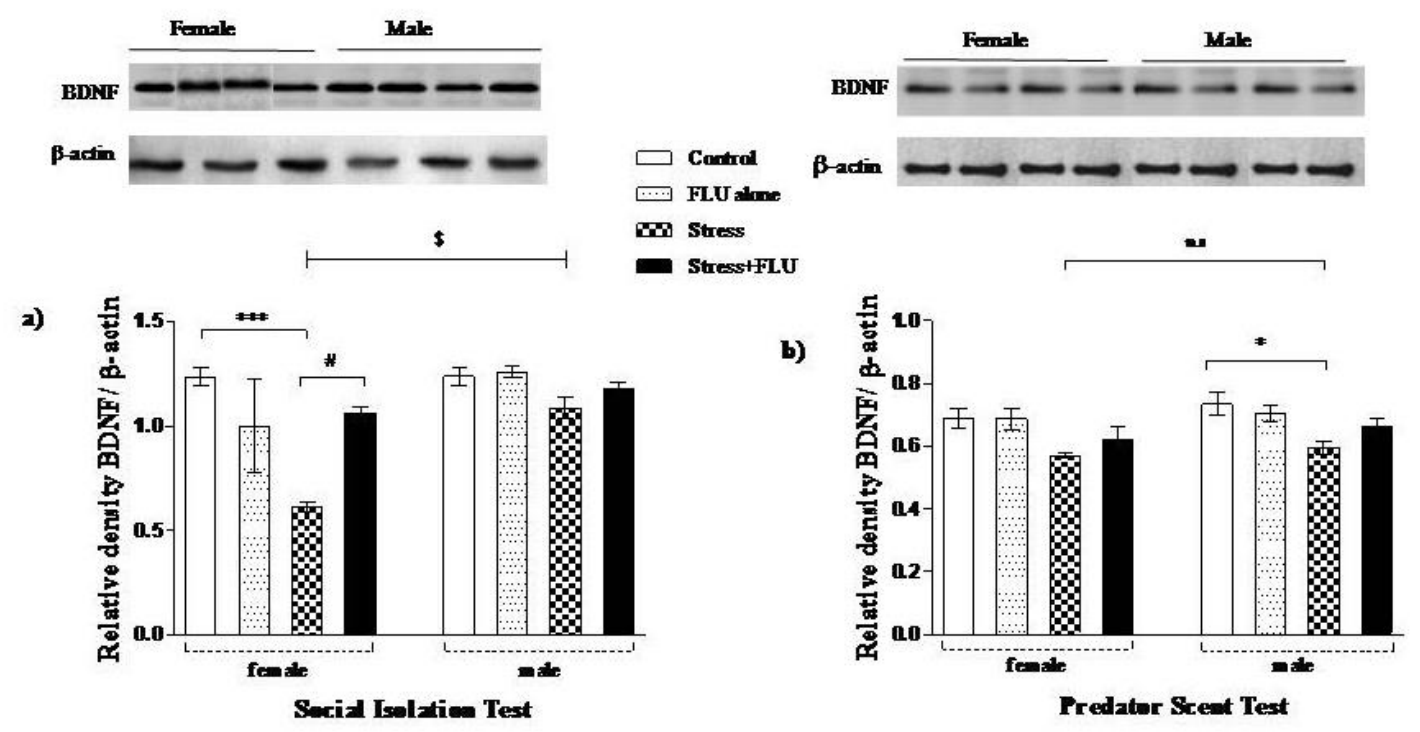

Figure 4. The effects of social isolation test (SIT, a) and predator scent tests (PST, b) on brain derived neurotrophic factor $(\mathrm{BDNF})$ in the amygdala in the rats and effects of fluoxetine (FLU) treatment. Rats received i.p injection of FLU, 5 $\mathrm{mg} / \mathrm{kg} /$ day or saline for 1 month. Anxiety of the animals were evaluated by using elevated plus maze (EPM) before and on the last day of drug/saline treatment ( $n=8$ for each group). The representative photographs of membranes collected from immunoblotting experiments of rats subjected to SI (a) or PSTs (b) treated with or without FLU. For the immunoblotting analysis $n=4$ rats were used for each group and the analyses were performed as duplicate. $\beta$-actin was used to normalize the amount of protein loaded in each lane.

${ }^{*}, \mathrm{p}<0.05 ;{ }^{* * * *}, \mathrm{p}<0.001$ shows the difference between the stress groups and the control group. ${ }^{*}, \mathrm{p}<0.05$ shows the difference between stress and stress+FLU groups. $\$, p<0.05$ represent the difference between the sexes. The data are expressed as means \pm SEM. 


\section{DISCUSSION}

Although it is well established that women suffer depressive disorders more frequently than males, considerably less attention has been given to gender differences in the animal models of depression. In this study, SIT and PSTs exerted discriminatory effects on anxiety indexes in the male and female rats and altered BDNF levels differently in both genders. We also found that chronic FLU treatment exerted discriminatory effects on BDNF expression in the hippocampus, the frontal cortex and the amygdala in different sexes.

SIT and PSTs are well-documented methods for inducing depression-like syndrome, and PTSD, respectively, in rodents [24, 25]. In our study female rats displayed greater anxiety responses than male rats in both stress conditions. This difference could be due to a variety of factors. Differences in hormonal properties, an activity of the monoaminergic system, structural changes such as hippocampal atrophy and reduced cyclic AMP response element binding (CREB) protein expression/phosphorylation could be considered [26].

We found that in the SIT, the expression of cortical BDNF levels was lowered to about $40 \%$ of their corresponding control levels in both genders. Although FLU treatment effectively restored reduced anxiety scores towards control levels, BDNF expression of these animals was not increased to pre-stress levels in both sexes. This finding may suggest that anxiety- reducing effect of FLU is not mediated through cortical BDNF activity, but it may be due to other mechanisms, such as inhibition of serotonin reuptake which is the crucial mechanism of action of SSRIs in the treatment of depression. In the PST, female rats exhibited increased anxiety and also displayed reduced expression of cortical BDNF levels. However, male rats had increased anxiety and contrary to the females exhibited an increased cortical BDNF expression. This finding differentiates the response pattern of cortical BDNF expression upon exposure to SIT versus PST in the male rats. This increased cortical BDNF expression may be genetically encoded in the male rats, rather than in the females, since the males are more prone to be exposed to natural predators and this mechanism may initiate effective protective neuronal or humoral responses concomitant with increased anxiety. This finding may indicate that BDNF expression does not always have to be in parallel with anxiety in different genders. Although FLU effectively reduced anxiety indices in both tests and in both sexes, it did not increase cortical BDNF levels in the females. In the PST, after FLU treatment male rats had reduced anxiety scores but FLU treatment reduced increased BDNF expression towards normal. This difference may be explained by assuming cortical BDNF expression may not be directly involved in the development of depressive symptoms in the male rats.

In the SIT, the expression of hippocampal BDNF levels was similarly lowered in both genders. Although FLU treatment effectively restored anxiety scores towards control levels, hippocampal BDNF expression in female rats was increased to pre-stress levels, but not in the male rats. Female and male rats displayed similar patterns in the PST in their hippocampal BDNF expression. However, FLU treatment increased BDNF levels in the females, but not in the male rats. The reason for this discrepancy in the male rats may be due to an anxiety reducing effect of FLU is not solely mediated through hippocampal BDNF activity, but may be due to other mechanisms shared by other SSRIs. Hormonal and structural differences between female and male rats may also contribute to this discrepancy. In literature, acute immobilization in rats leads to stress causing a decrease in BDNF levels [5,27]. It has also been reported that stress-induced changes in the hippocampus may be due to development of depression and together with volume decrease and the expression of BDNF levels is decreased. Types of stress such as social isolation, immobilization, restraint, and footshock decrease the expression of BDNF in the hippocampus and FLU increases hippocampal BDNF levels in some studies $[2,14,15]$. Our results indicate that there is a definite response to FLU treatment for both social isolation and predator stress groups regarding hippocampal BDNF levels.

In the SIT, female rats displayed reduced BDNF levels markedly in the amygdala when compared with other brain regions, and FLU treatment normalized this reduction concomitantly with its antidepressant effect. In the same test, in the male rat's stressor did not reduce BDNF levels in the amygdala and FLU treatment did not alter neurotrophin levels. PST was found to be less effective in reducing BDNF levels in the amygdala in both genders. Examination of BDNF expression in the amygdala in PST may not be suitable for evaluation in rat models of depression. These findings may indicate that alteration of BDNF expression to stressful conditions in the amygdala is less prominent than that observed in the frontal cortex and hippocampus.

\section{CONCLUSION}

Our findings may appear to be partially contrary to the neurotrophin hypothesis of depression, but may implicate that alteration of BDNF levels in the cortical structures does not play a critical role in the pathophysiology of depression in rat models. Preclinical and clinical studies have focused on mood disorders 
such as stress and depression and its effects on different brain regions as hippocampus, amygdala and frontal cortex. Moreover, hippocampus has connections with frontal cortex and amygdala, both are contributed to regulation of cognition and emotional states [2, 27, 28]. Imaging studies have shown that hippocampal volume is decreased in those patients with mood disorders [29, 30]. It is known that SSRIs increase the expression of BDNF in the brain and this effect is related to the duration of antidepressant drug therapy [2, 15]. Antidepressants have been effective in alleviating depressive mood but have been less effective in decreasing the symptoms of re-experiencing the fear conditions.

Our findings indicate that sex differences must be considered in studies related to mood disorders of animal models suggesting that BDNF expression in different brain regions are altered differentially in a gender-dependent manner in rats. Antianxiety effect of FLU appears to be not mediated through increasing BDNF activity in the cortex in both genders. Increased BDNF expression in hippocampus and amygdala may reflect an antidepressant effect of FLU in the female rats, but not in the males.

\section{MATERIALS AND METHODS}

\subsection{Animals and Conditions}

After the approval from the Institutional Ethical Committee of Marmara University was obtained for this study, all experiments and treatment procedures were initiated (MÜHDEK approval no: 86.2013.mar). Sprague-Dawley rats of both sexes weighing 180-200 g supplied from Marmara University Animal Center (DEHAMER) were used in the study. The rats were sheltered to the housing conditions for 10 days with at $21 \pm 3{ }^{\circ} \mathrm{C}$ and $50 \pm 10 \%$ humidity and reversed $12 \mathrm{~h}$ light/dark cycle with ad libitum and with access to standard rat chow and water. All experiments were carried out in the dark phase using a dim light source.

\subsection{Stress Tests}

For experimental design random selection was performed to construct control, FLU alone, stress, stress+FLU treatment groups for each stress tests after 10 days of acclimation period. The same procedure was repeated for both male and female rats. Total 128 rats were used including eight rats per group.

\subsubsection{Predator scent test}

The stress condition was produced by placing the rats in a plexiglass $30 \times 30 \times 40 \mathrm{~cm}$ sized cage on 125 $\mathrm{ml}$ of dirty cat litter for $10 \mathrm{mins}$. The cat litter had been used for 2 days by the same cat and had been sifted for stools. The control animals were placed in an identical cage with fresh, unused litter for 10 mins [31-33]. The rats were subjected to clean cat litter as a situational reminder 1 month after the onset of the stress. Anxiety indices were calculated using elevated plus maze (EPM) setup on the first and last days.

\subsubsection{Social isolation test}

The stress condition was applied by placing only one rat for each $30 \times 30 \times 20 \mathrm{~cm}$ sized cage for all groups. Each animal was kept for a habituation period for one month. Anxiety indices were calculated using EPM setup on the first and last days.

\subsection{Drugs and Solutions}

For both stress conditions, treatment groups received intraperitoneal (i.p) injections of $5 \mathrm{mg} / \mathrm{kg}$ FLU (Eli Lilly, Turkey) suspended in physiological saline, for 1 month. Control groups received physiological saline injections. The injections were applied $10 \mathrm{mins}$ before the test, and continued daily, at the same time of the day.

\subsection{EPM Experiments}

The rats exposed to PST were placed on an EPM for 5mins, immediately after that they were subjected to stituation reminder. However, for SIT, rats were placed on the EPM after the injections. The EPM had two opposing closed $(50 \mathrm{~cm} \times 10 \mathrm{~cm})$ and two opposing open $(50 \mathrm{~cm} \times 10 \mathrm{~cm})$ arms. The closed arms were surrounded by $10 \mathrm{~cm}$ high opaque walls on three sides. The height of EPM was $50 \mathrm{~cm}$ from the ground. The maze was cleaned with $5 \%$ alcohol solution before the rats were placed on EPM. Each rat was placed in the central square of the EPM facing towards one of the open arms. The behavioral experiments were recorded during $5 \mathrm{mins}$ using a video camera placed on top of the setup and recordings were observed to score behavioral parameters. An arm entry was defined as an animal entering the arm with all four feet and the number of entries into open and enclosed arms were scored $[34,35]$. The anxiety indices $\left(\mathrm{N}_{\text {anxiety }}\right)$ was calculated by using A (cumulative 
time spent in open arms (s)), B (open arm entries), $\mathrm{C}$ (total arm entries) parameters and the formula: $\mathrm{N}_{\text {anxiety }}=$ $1-1 / 2[(\mathrm{~A} / 300 \mathrm{~s})+(\mathrm{B} / \mathrm{C})]$

\subsection{Tissue Collection, Sample Preparation and Immunoblotting}

Following decapitation, brains were removed, the hippocampus, the frontal cortex and the amygdala were dissected by the Rat Brain Atlas [36]. Obtained tissues were stored at $-80^{\circ} \mathrm{C}$ for immunoblotting. The frozen tissues were weighed and homogenized in $10 \mathrm{mM}$ Tris- $\mathrm{HCl}(\mathrm{pH}$ 7.2) buffer containing $1 \mathrm{mM}$ EDTA and protease inhibitors including $0.2 \mathrm{mM}$ PMSF, $1 \mu \mathrm{g} / \mathrm{ml}$ leupeptin, $1 \mu \mathrm{M}$ pepsitatin, $10 \mu \mathrm{g} / \mathrm{ml}$ soybean trypsin inhibitor with Ultraturrax homogenizer. Samples from each region of every rat were processed and centrifuged at $4{ }^{\circ} \mathrm{C}$ at $300 \times \mathrm{g}$ for 5 mins. Obtained from first centrifuge supernatant was centrifuged again at $13.200 \times \mathrm{g}$ for 90 mins. The protein content was determined by Lowry method [37]. Hundred micrograms of protein was loaded onto $12 \%$ sodium dodecyl sulfate-polyacrylamide gels and electrophoretically transferred onto nitro-cellulose membranes $(0.45 \mu \mathrm{m}$, Schleicher and Schuell, Germany) for 90 mins at $80 \mathrm{~V}$. The membranes were blocked with tris-buffered saline (TBS) containing 1\% bovine serum albumin and $0.05 \%$ Tween-20 at room temperature for $1 \mathrm{~h}$ and incubated overnight at $4^{\circ} \mathrm{C}$ with the primary antibodies against BDNF (1:200 each). The BDNF specific antibodies and secondary antibodies were supplied by Santa Cruz Biotechnology, Inc. (Santa Cruz, CA, USA). The blots were washed three times with TBS containing $0.05 \%$ Tween-20 and incubated with alkaline phosphatase-conjugated secondary antibodies for 60 mins at $21 \pm 3{ }^{\circ} \mathrm{C}$ temperature. Nitroblue tetrazolium/ 5-bromo-4-chloro-3-indole phosphate was used to detect the antibodyantigen complex. For the immunoblotting analysis $n=4$ rats were used for each group and the analyses were performed in duplicate. The densitometric analyses were performed with Bio-Rad Molecular Analyst software.

\subsection{Statistics}

Statistical analysis was carried out using GraphPad Prism (GraphPad Software, San Diego; CA; USA). The data were presented as means \pm SEM. To analyze anxiety indices in the treatment groups, two-way analysis of variance followed by Bonferroni post hoc test was used. Values of $p<0.05$ were considered significant for all statistical calculations. One-way analysis of variance followed by Tukey's multiple comparison tests was used for the comparison of the immunoblotting data.

Acknowledgements: This study was supported by a grant from Istanbul Medeniyet University Fund (Project number: TSA-2013-280).

Author contributions: Concept -A.A., S.O., R.O.; Design - A.A., S.O.; Supervision -A.A.; Resource -S.O., A.A; Materials - A.A., S.O.; Data Collection and/or Processing - A.A., S.O., R.O.; Analysis and/or Interpretation - A.A., R.O.; Literature Search - A.A., S.O., R.O.; Writing - A.A., R.O.; Critical Reviews - A.A., S.O., R.O.

Conflict of interest statement: The authors declare no conflict of interest.

\section{REFERENCES}

[1] Duman RS. Pathophysiology of depression: the concept of synaptic plasticity. Eur Psychiatry. 2002; 3: 306-310.

[2] Duman RS, Monteggia LM. A Neurotrophic Model for Stress-Related Mood Disorders Biol Psychiatry. 2006; 59(12):1116-1127.

[3] Khundakar AA, Zetterström TS. Biphasic change in BDNF gene expression following antidepressant drug treatment explained by differential transcript regulation. Brain Res. 2006; 1106(1): 12-20.

[4] Shimizu E, Hashimoto K, Okamura N, Koike K, Komatsu N, Kumakiri C, Nakazato M, Watanabe H, Shinoda N, Okada S, Iyo M. Alteration of serum levels of brain-derived neurotrophic factor (BDNF) in depressed patients with or without antidepressants. Biol Psychiatry. 2003; 54: 70-75.

[5] Smith MA, Makino S, Kvetnansky R, Post RM. Stress alters the express brain derived neurotrophic factor and neurotrophin-3 mRNAs in the hippocampus. J Neurosci. 1995; 15: 1768-1777.

[6] Nibuya M, Takahashi M, Russell DS, Duman RS. Repeated stress increases catalytic TrkB mRNA in rat hippocampus. Neurosci Lett. 1999; 267(2): 81-84. 
[7] Roceri M, Cirulli F, Pessina C, Peretto P, Racagni G, Riva MA. Postnatal repeated maternal deprivation produces agedependent changes of brain-derived neurotrophic factor expression in selected rat brain regions. Biol Psychiatry. 2004; 55(7): 708-714.

[8] Kozlovsky N, Matar MA, Kaplan Z, Kotler M, Zohar J, Cohen H. Long-term down-regulation of BDNF mRNA in rat hippocampal CA1 subregion correlates with PTSD-like behavioural stress response. Int J Neuropsychopharmacol. 2007; 10(6): 741-758.

[9] Taliaz D, Loya A, Gersner R, Haramati S, Chen A, Zangen A. Resilience to chronic stress is mediated by hippocampal brain-derived neurotrophic factor. J Neurosci. 2011; 31(12): 4475-4483.

[10] Martinotti G, Sepede G, Brunetti M, Ricci V, Gambi F, Chillemi E, Vellante F, Signorelli M, Pettorruso M, De Risio L, Aguglia E, Angelucci F, Caltagirone C, Di Giannantonio M. BDNF concentration and impulsiveness level in posttraumatic stress disorder. 2015; 30; 229(3): 814-818.

[11] Hashimoto K. Brain-derived neurotrophic factor (BDNF) and its precursor proBDNF as diagnostic biomarkers for major depressive disorder and bipolar disorder. Eur Arch Psychiatry Clin Neurosci. 2015; 265(1): 83-84.

[12] Shirayama Y, Chen AC, Nakagawa S, Russell RS, Duman RS. Brain derived neurotrophic factor produced antidepressant effects in behavioral models of depression. J Neurosci. 2002; 22: 3251-3261.

[13] Siuciak JA, Boylan C, Fritsche M, Altar CA, Lindsay RM. BDNF increases monoaminergic activity in rat brain following intracerebroventricular or intraparenchymal administration. Brain Res. 1996; 710: 11-20.

[14] Nibuya M, Nestler EJ, Duman RS. Chronic antidepressant administration increases the expression of cAMP response element binding protein (CREB) in rat hippocampus. Neurosci 1996; 16: 2365-2372.

[15] Copella AL, Pei Q, Zetterström TSC. Bi-phasic change in BDNF gene expression following antidepressant drug treatment. Neuropharmacol. 2003; 44: 903-910.

[16] Duman RS, Malberg J, Nakagawa S, D'Sa C. Neuronal plasticity and survival in mood disorders. Biol Psychiatry. 2000; 48(8): 732-739.

[17] Kang HJ, Adams DH, Simen A, Simen BB, Rajkowska G, Stockmeier CA, Overholser JC, Meltzer HY, Jurjus GJ, Konick LC, Newton SS, Duman RS. Gene Expression Profiling in Postmortem Prefrontal Cortex of Major Depressive Disorder. J Neurosci. 2007; 27(48): 13329-13340.

[18] Mendez-David I, Tritschler L, Ali ZE, Damiens MH, Pallardy M, David DJ, Kerdine-Römer S, Gardier AM. Nrf2signaling and BDNF: A new target for the antidepressant-like activity of chronic fluoxetine treatment in a mouse model of anxiety/depression. Neurosci Lett. 2015; 597: 121-126.

[19] Carter-Snell C, Hegadoren K. Stress disorders and gender: implications for theory and research. Can J Nurs Res. 2003; 35: 34-55.

[20] Kessler R. Epidemiology of women and depression. J Affect Disord. 2003; 74(1): 5-13.

[21] Lin Y, Ter Horst GJ, Wichmann R, Bakker P, Liu A,Li X, Westenbroek C. Sex differences in the effects of acute and chronic stress and recovery after long-term stress on stress-related brain regions of rats. Cereb Cortex. 2009; 19(9): 1978-1989.

[22] Franklin TB, Perrot-Sinal TS. Sex and ovarian steroids modulate brain-derived neurotrophic factor (BDNF) protein levels in rat hippocampus under stressful and non-stressful conditions. Psychoneuroendocrinology. 2006; 31(1): 3848.

[23] Mitic M,Simic I, Djordjevic J, Radojcic MB, Adzic M. Gender-specific effects of fluoxetine on hippocampal glucocorticoid receptor phosphorylation and behavior in chronically stressed rats. Neuropharmacology. 2013; 70: $100-611$.

[24] Leser IN, Wagner S. The effects of acute social isolation on long-term social recognition memory. Neurobiol Learn Mem. 2015; 124: 97-103.

[25] Zlatkovic J, Bernardi RE, Filipovic D. Protective effect of Hsp70i against chronic social isolation stress in the rat hippocampus. J Neural Transm. 2014; 121: 3-14.

[26] Nestler EJ, Barrett M, DiLeone RJ, Eisch AJ, Gold SJ, Monteggia LM. Neurobiology of depression. Neuron. 2002; 34: $13-25$.

[27] McEwen B. Stress and hippocampus plasticity. Curr Opin Neurobiol. 1999; 5:205-216.

[28] Duman RS, Malberg J, Thome J. Neural Plasticity to stress and antidepressant treatment. Biol Psychiatry. 1999; 46: 1181-1191. 
[29] Sapolsky R. Glucocorticoids and atrophy of the human hippocampus. Sci. 1999; 273: 749-750.

[30] Sapolsky R. Depression, antidepressant, and the shrinking hippocampus. Proc Natl Acad Sci USA. 2001; 98 : 1232012322.

[31] Zohar J, Matar MA, Ifergane G, Kaplan Z, Cohen H. Brief post-stressor treatment with pregabalin in an animal model for PTSD: Short-term anxiolytic effects without long-term anxiogenic effect. Eur Neuropsychopharmacol. 2008; 9: 653-666.

[32] Cohen H, Matar MA., Richter-Levin G, Zohar J. The contribution of an animal model toward uncovering biological risk factors for PTSD. Ann N Y Acad Sci. 2006; 1071: 335-350.

[33] Mazor A, Michael A, Matar A, Kaplan Z, Kozlovsky N, Zohar J, Kaplan Z, Cohen H. Gender-related qualitative differences in baseline and post-stress anxiety Responses are not reflected in the incidence of criterion-based PTSDlike behaviour patterns. World J Biol Psychiatry. 2009; 10: 856-869.

[34] Pellow S, Chopin P, File SE, Briley M. Validation of open-closed arm entries in an elevated plus maze as measure of anxiety in the rat. J Neurosci Methods. 1985; 14(3): 149-167.

[35] Cohen H, Matar MA, Zohar J. Animal Models of Post-Traumatic Stress. Preclinical Models of Neurologic and Psychiatric Disorders. Curr Protoc Neurosci. 2013; 9(45 Suppl 64):1-18.

[36] Paxinos G, Watson C. The rat brain in stereotaxic coordinates. 6nd ed. London:Academic Press, 1986.

[37] Lowry OH, Rosebrough NJ, Farr AL, Randall RJ. Protein measurement with the Folin phenol reagent. J Biol Chem. 1951; 193(1): 265-275. 